\title{
Estudo da coordenação de relés de sobrecorrente e modelagem de proteção adaptativa considerando Geração Distribuída em Sistemas de Distribuição
}

\author{
Luis H. P. Vasconcelos*. Aryfrance R. Almeida**. Nelber X. Melo*** \\ José G. S. Carvalho ${ }^{* * * * *}$. Bartolomeu F. dos Santos, Jr. ${ }^{* * * * * *}$ \\ * Departamento de Engenharia Elétrica, Universidade Federal do Piauí, \\ Piauí, Brasil, (Tel: (86)99525-8906; e-mail: eng.luishenrique14@hotmail.com). \\ ** Departamento de Engenharia Elétrica, Universidade Federal do Piauí, \\ Piauí, Brasil, (Tel: (86)99935-6284; e-mail: aryfrance@ ufpi.edu.br). \\ *** Departamento de Engenharia Elétrica, Universidade Federal do Piauí, \\ Piauí, Brasil, (Tel: (86)98820-8245; e-mail: nelber@ufpi.edu.br). \\ **** Departamento de Engenharia Elétrica, Universidade Federal do Piauí, \\ Piauí, Brasil, (Tel: (86)99534-1203; e-mail: eng.genilson@hotmail.com). \\ ***** Departamento de Engenharia Elétrica, Universidade Federal do Piauí, \\ Piauí, Brasil, (Tel: (86)99801-8178; e-mail: bartolomeuf@ufpi.edu.br).
}

\begin{abstract}
There is a growing increase in the use of Distributed Generation based on alternative sources to supply the population's electricity consumption. However, in spite of the many advantages that this practice provides, there are some precautions that must be taken, especially with regard to the protection aspect of the transmission and distribution systems. This work accomplishes the verification of the impacts that the insertion of Distributed Generation can cause in the coordination of the overcurrent relays and a model of relay with adaptive adjustment to minimize these effects. Initially, a 13-bus IEEE standard distribution system was modeled in the ATPDraw software with all its main elements as source, loads, transmission lines and power transformers. Then, two coordinated cascaded overcurrent relays were added to the system and their respective actuation times and coordinating margins were obtained for minimum and maximum short-circuit currents. Finally, an adaptive protection method has been proposed to adapt relay coordination for several scenarios, in which a control block receives as parameters the maximum and minimum short circuit currents for each topology and modifies the downstream relay setting. as needed while maintaining selectivity and speed of protection.
\end{abstract}

\begin{abstract}
Resumo: Existe um crescente aumento do uso da Geração Distribuída com base em fontes alternativas para suprir o consumo de energia elétrica da população. No entanto, apesar das diversas vantagens que essa prática proporciona, existem alguns cuidados que devem ser tomados, principalmente no que diz respeito ao aspecto da proteção dos sistemas de transmissão e distribuição de energia. Este trabalho realiza a verificação dos impactos que a inserção de Geração Distribuída pode causar na coordenação dos relés de sobrecorrente e uma modelagem de relé com ajuste adaptativo para minimizar esses efeitos. Inicialmente, foi realizada a modelagem de um sistema de distribuição padrão do IEEE de 13 barras no software ATPDraw com todos os seus elementos principais como fonte, cargas, linhas de transmissão e transformadores de potência. Em seguida, foram adicionados ao sistema dois relés de sobrecorrente em cascata coordenados e obtidos seus respectivos tempos de atuação e margens de coordenação para correntes de curto-circuito mínima e máxima. Os mesmos testes foram realizados considerando a operação de gerações distribuídas conectadas em alguns pontos do sistema. Por fim, foi proposto um método de proteção adaptativa capaz de adequar a coordenação dos relés para diversos cenários, na qual um bloco de controle recebe como parâmetros as correntes de curto-circuito máxima e mínima para cada topologia e modifica o ajuste do relé a jusante conforme necessário, mantendo a seletividade e a velocidade da proteção.
\end{abstract}

Keywords: Distributed generation; coordination; relays; adaptive protection; ATPDraw.

Palavras-chaves: Geração Distribuída; coordenação; relés; proteção adaptativa; ATPDraw.

\section{INTRODUÇÃO}

A principal função do Sistema Elétrico de Potência (SEP) é fornecer energia elétrica aos seus consumidores de forma confiável e econômica. Devido ao alto custo dos equipamentos que compõem um SEP, este representa um investimento considerável de capital. Por isso, para o retorno sobre este investimento ser maximizado, a margem de utilização do sistema deve ser a maior possível. No entanto, essa margem deve respeitar os limites de segurança para operação de um sistema elétrico confiável. A confiabilidade de um sistema de energia está diretamente relacionada com o desempenho de seus esquemas de proteção. Um sistema de 
proteção eficiente deve possuir cinco características essenciais, são elas: sensibilidade, velocidade, seletividade, estabilidade e confiabilidade (Rush, 2011).

O modelo de sistema elétrico baseado em uma única fonte de energia, para o qual o esquema de proteção contra curtoscircuitos foi projetado vem sendo rapidamente alterado pela conexão de unidades de Geração Distribuída (GD). Essa mudança de paradigma representa um desafio para a forma tradicional de eliminar falhas do sistema e, portanto, requer uma análise apurada dos impactos causados por essas conexões a fim de validar a adoção de novas filosofias de proteção pelas distribuidoras (Luiz, 2012). Alguns resultados de estudos sobre o efeito da inserção de unidades de GD no desempenho dos equipamentos de proteção são discutidos em trabalhos como Dugan and McDermott (2002), Britto et al. (2004) e Thong et al. (2007).

Como possibilidade de solução, a proteção adaptativa é uma filosofia que permite fazer ajustes no sistema de proteção para torná-lo mais adequado às condições variáveis do sistema elétrico. Os relés atuais permitem a configuração de vários conjuntos de ajustes de proteção, chamados de grupos de ajustes, que podem corresponder a diferentes situações operacionais ou topológicas às quais a rede de distribuição pode ser submetida (Luiz, 2012). Em trabalhos como Cheung et al. (2008), Yongli et al. (2009) e Chaves and Leão (2014), os autores propõem estratégias de proteção adaptativa capazes de alterar os ajustes dos equipamentos de proteção de acordo com a configuração do sistema, tornando o sistema de proteção mais eficiente.

Este documento tem como objetivo analisar os efeitos da geração distribuída para a coordenação dos relés de sobrecorrente em um sistema de distribuição, além de propor um método de ajuste adaptativo que utiliza os estados dos disjuntores das GDs para determinar a configuração de operação do sistema elétrico e, a partir das correntes de falta correspondentes de cada situação, calcula o ajuste adequado. Assim, é possível manter a seletividade e a velocidade da proteção independente da topologia de operação do sistema.

\section{METODOLOGIA}

O software de simulação utilizado neste trabalho foi o ATPDraw, um dos programas mais usados na área acadêmica para estudos de distúrbios em sistemas elétricos (Miguel 2011). Primeiramente, realizou-se a modelagem do sistema de distribuição modelo do IEEE de 13 barras (Kersting, 1991). Em seguida, foram inseridos, nas barras 650 e 632, dois relés de sobrecorrente, coordenados de modo a obedecer aos critérios de seletividade. Então, foram obtidos seus tempos de atuação (td R1 e td R2) e suas margens de coordenação $(\Delta \mathrm{t})$ para as seguintes faltas: bifásica na barra 680, no qual a margem de coordenação é máxima, e trifásica no início da linha L632- 671, no qual a margem de coordenação é mínima. Todas as faltas neste trabalho foram aplicadas em $0,1 \mathrm{~s}$ de simulação.

Os testes foram realizados também para os casos com a GD inserida em duas localizações distintas (barras 650 e 632). Foram calculados, para os casos com GD, as diferenças percentuais das margens de coordenação (Dif. $\Delta \mathrm{t}$ ) com relação ao caso sem GD. Por fim, foi modelado um bloco de controle responsável por alterar o ajuste do relé R1 (dial de tempo) de acordo com a presença da GD no sistema. Então, foram realizados novos testes utilizando o bloco de ajuste adaptativo e comparados os resultados para validação do método proposto.

\subsection{Modelagem do sistema elétrico no ATPDraw}

O circuito utilizado neste estudo corresponde ao sistema de distribuição padrão IEEE de 13 barras. A Fig. 1 apresenta o diagrama do circuito com as suas respectivas barras enumeradas e as indicações dos pontos de conexão das GDs.

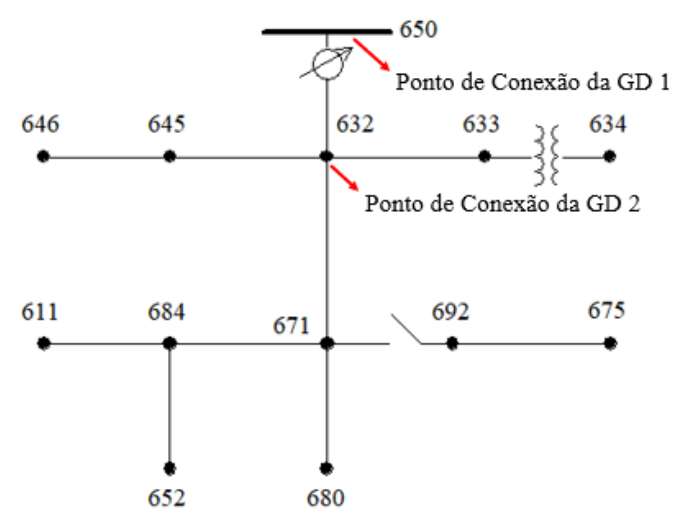

Fig. 1 Diagrama do sistema padrão IEEE de 13 barras.

As duas GDs utilizadas neste trabalho possuirão características exatamente iguais. $\mathrm{O}$ tipo de gerador distribuído utilizado é uma máquina síncrona, que pode ser modelada simplesmente com uma fonte tensão e uma reatância (sub-transitória) em série. Um valor típico da reatância sub-trasitória para geradores de pequeno porte é 0,2 pu, calculados na base de $480 \mathrm{~V}$ e 100 MVA (Kinderman, 2010). A potência instalada da GD é de $300 \mathrm{~kW}$ e foi fixada através do bloco LF_UP do ATPDraw. O transformador de conexão da GD utilizará ligação Delta-Estrela aterrado, pois é a forma de conexão mais adotada no caso de geração própria (Luiz, 2012). O componente TACS FORTRAN1 controlará a abertura e o fechamento do disjuntor da GD $(0=$ aberto e $1=$ fechado). A Fig. 2 ilustra a modelagem da GD no ATPDraw.

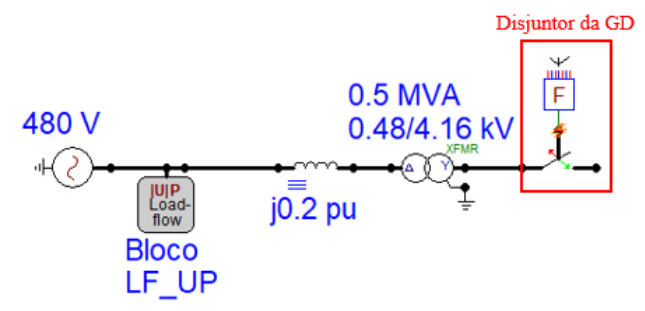

Fig. 2 Geração Distribuída modelada no ATPDraw.

Os relés foram modelados através do componente W1RELAY51I do ATP que tem como parâmetros de entrada o dial de tempo, a corrente de pick-up e os valores das constantes A, B e Q que dependem do tipo de curva ao qual deseja- se utilizar. Por exemplo, para a curva IEC extremamente inversa (utilizada neste trabalho), temos: $\mathrm{A}=$ $80, \mathrm{~B}=0$ e $\mathrm{Q}=2$. Em conjunto com o relé, foi utilizado o 
componente MODELS ABC2RMS para fornecer o valor RMS da corrente no ponto de instalação do relé e os elementos TACS para acionamento do disjuntor conforme ilustrado na Fig. 3.

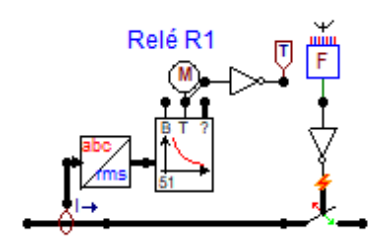

Fig. 3 Conjunto relé e disjuntor no ATPDraw.

Na Fig. 4 é ilustrado todo o sistema modelado no ATPDraw.

\subsection{Parametrização dos relés sem a GD inserida no sistema.}

O primeiro relé (R1) localiza-se no início da linha L650-632, de forma a proteger a mesma e funcionar como relé de retaguarda do relé $\mathrm{R} 2$ para trechos mais à jusante. O relé R2 localiza-se logo após a barra 632 e antes da linha L632-671, protegendo desde o ponto de sua instalação até o final do alimentador. A curva de atuação escolhida para os relés foi a IEC extremamente inversa, por ser a mais indicada para sistemas de distribuição primária (Filho and Mamede, 2011). Esta curva obedece a seguinte equação:

$$
\mathrm{td}=\frac{80 x D T}{\left(\frac{\text { ICC }}{\text { Iaj }}\right)^{2}-1}
$$

Onde td é o tempo de disparo do relé (s); DT é o dial de tempo (s); Icc é a corrente de curto-circuito (A); e Iaj é a corrente de ajuste do relé (A).

Inicialmente parametrizou-se o relé R2 conforme os critérios abordados em (Rush, 2011). O ajuste de corrente deve ser escolhido de forma que o relé não opere para a corrente de carga máxima no circuito protegido, mas que opere para uma corrente igual ou maior à corrente de falta mínima esperada. Como não existe nenhum dispositivo de proteção à jusante de R2 para coordenar, foi escolhido um dial de tempo (DTR2) arbitrário baixo de 0,1 para se trabalhar com valores baixos de tempo de simulação.

A parametrização de R1 foi feita de forma a garantir a coordenação com R2. De acordo com Filho and Mamede (2011), para garantir a seletividade da proteção, deve-se manter uma diferença mínima de $0,4 \mathrm{~s}$ entre os tempos de operação de dois relés em cascata, levando em consideração o tempo de operação do disjuntor, a tolerância do fabricante do disjuntor e o tempo de segurança do projeto. Em Rush (2011), a margem típica de coordenação entre dois relés é de 0,3 s para relés numéricos (neste estudo adotou-se 0,3 s). Após alguns testes, concluiu-se que as curvas dos relés aproximam- se, o que significa que a margem de atuação mínima deve ser estabelecida para a corrente de falta máxima percebida pelo relé mais à jusante (R2), que corresponde a uma falta trifásica no início da linha L632-671. Sendo assim, o tempo de atuação de R1 para a falta máxima detectada por $\mathrm{R} 2$ deve ser dado pala seguinte equação:

$$
\text { tdR1(Iccmáx) } \geq \text { tdR2(Iccmáx) + 0,3 s }
$$

Onde o tdR1(Iccmáx) é o tempo de disparo de R1 (s) para a falta máxima medida por R2 e tdR2(Iccmáx) é o tempo de disparo de R2 (s) para a falta máxima medida por R2. Como ambos os relés utilizarão a curva IEC extremamente inversa, substituindo (1) em (2), o dial de tempo mais adequado para o relé $\mathrm{R} 1$ deve ser dado por:

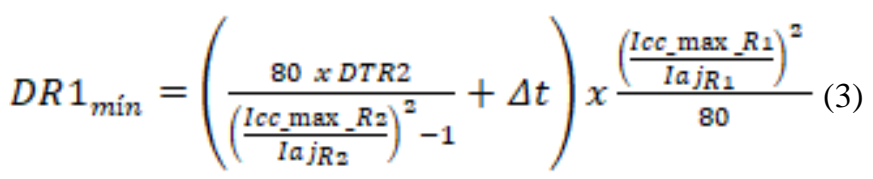

Onde DR1mín é o dial de tempo mínimo para o relé R1 (s); DTR2 é o dial de tempo do relé R2 (s); Icc_max_R1 é a corrente de curto-circuito máxima detectada por R1 (A); Icc_max_R2 é a corrente de curto-circuito máxima detectada por R2 (A); IajR2 é a corrente de ajuste de R2 (A); e $\Delta t$ é a margem de coordenação mínima (s).

Após seguir esses procedimentos de cálculos, foram utilizados os ajustes dos relés apresentados na Tabela 1.

\section{Tabela 1. Ajustes dos relés (sem GD em operação)}

\begin{tabular}{|c|c|c|}
\hline Parâmetros & R1 & R2 \\
\hline Corrente de ajuste (A) & 675 & 514 \\
\hline Dial de Tempo (s) & 0,17 & 0,1 \\
\hline Tipo de Curva & EI & EI \\
\hline
\end{tabular}

\subsection{Modelagem do Controle de ajuste adaptativo.}

A proteção adaptativa foi realizada a partir da criação de um componente em linguagem MODELS, denominado Controle. Esse bloco recebe como entradas os estados dos disjuntores das GDs $(0=$ aberto e $1=$ fechado $)$ e fornece como saída o valor do dial de tempo apropriado para a nova topologia do sistema que será utilizado pelo relé R1. Esse valor deve ser calculado de acordo com (3). O reajuste do relé consite na alteração somente do seu valor de dial de tempo, o que significa movimentar verticalmente a sua curva de atuação. Assim, para o caso em que a presença das GDs cause diminuição nas margens de coordenação, o reajuste do relé R1 aumentará a margem de coordenação mínima para valores adequados acima do critério de segurança $(0,3 \mathrm{~s})$, restaurando a característica de seletividade da proteção.

Por outro lado, caso a presença da GD aumente as margens de coordenação, o reajuste do relé $\mathrm{R} 1$ diminuirá essas margens e tornará sua atuação de retaguarda mais veloz. A presença de GD no sistema altera seus níveis de correntes de curto-circuito (Choi et al. 2008), portanto, o bloco de controle criado recebe como parâmetros de entrada as correntes de falta máximas detectadas pelos relés em cada caso, além das suas correntes de ajustes e do dial de tempo do relé R2, previamente calculados. 


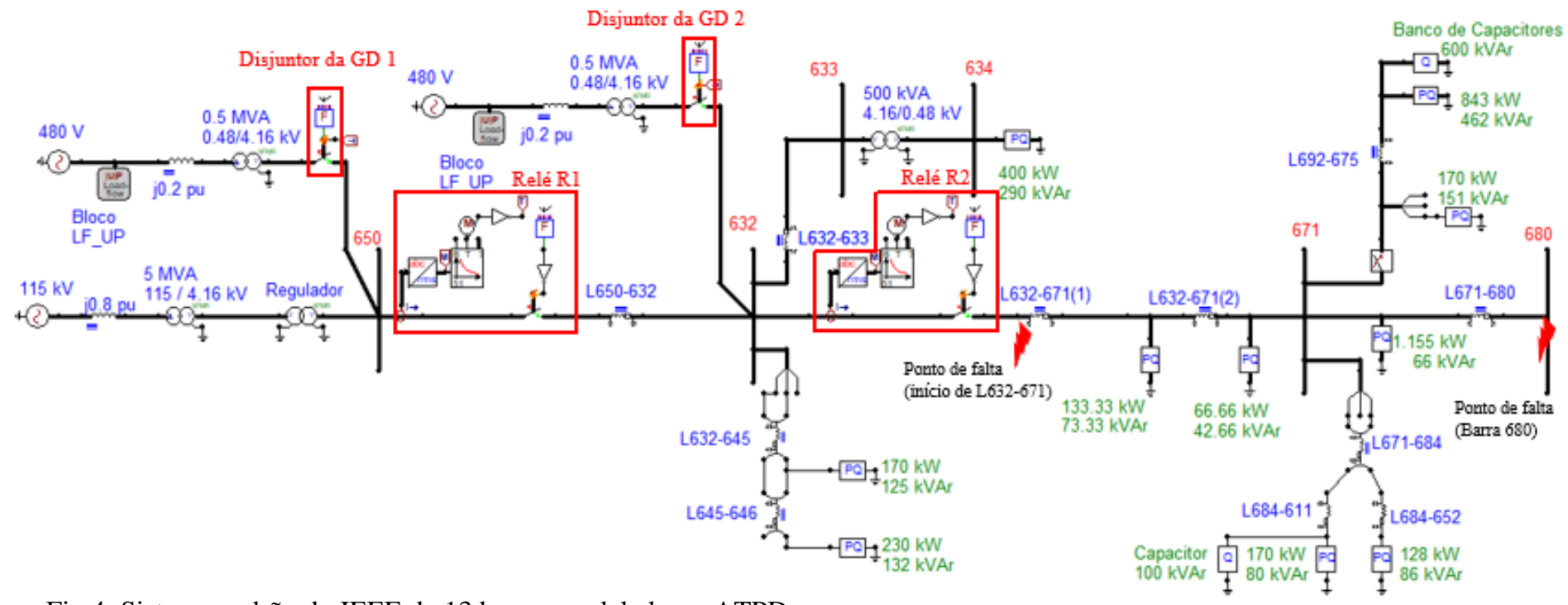

Fig 4. Sistema padrão do IEEE de 13 barras modelado no ATPDraw.

A Fig. 5 ilustra o Bloco de Controle para ajuste adaptativo modelado no ATPDraw. A Tabela 2 mostra o valor do dial de tempo fornecido pelo bloco de controle para cada caso, conforme os estados dos disjuntores das GDs.

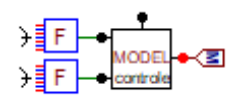

Fig. 5 Bloco de Controle para ajuste adaptativo.

Tabela 2. Ajuste adaptativo do relé R1

\begin{tabular}{|c|c|c|}
\hline \multicolumn{2}{|c|}{ Estado Disjuntor } & \multirow{2}{*}{ DR1 } \\
\hline GD 1 & GD 2 & 0,17 \\
\hline 0 & 0 & 0,14 \\
\hline 0 & 1 & 0,23 \\
\hline 1 & 0 & 0,2 \\
\hline 1 & 1 & \\
\hline
\end{tabular}

A Fig. 6 mostra a curva do relé R2 (dial fixo) e as curvas do relé R1 para os diferentes valores de dial mostrados na Tabela 2. Optou-se por limitar o eixo horizontal para valores de corrente dentro do intervalo de 2000 a 7000 A para melhor visualização das curvas.

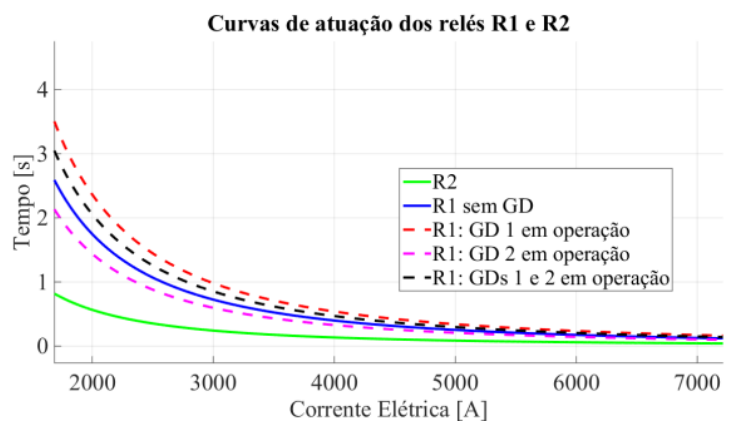

Fig 6. Curvas de atuação dos relés R1 e R2.

\section{RESULTADOS E DISCUSSÕES}

As Figs. 7 e 8 apresentam os valores RMS das correntes na fase A medidas pelos relés em cada caso com faltas aplicadas no início da linha L632-671 (falta máxima).

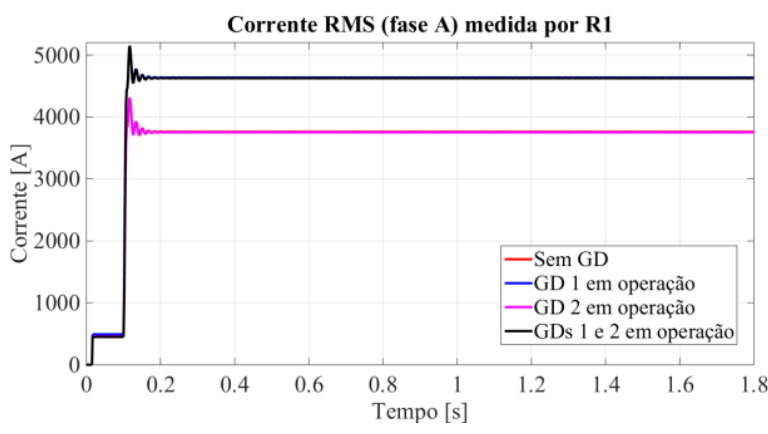

Fig 7. Corrente RMS na fase A medida por R1.

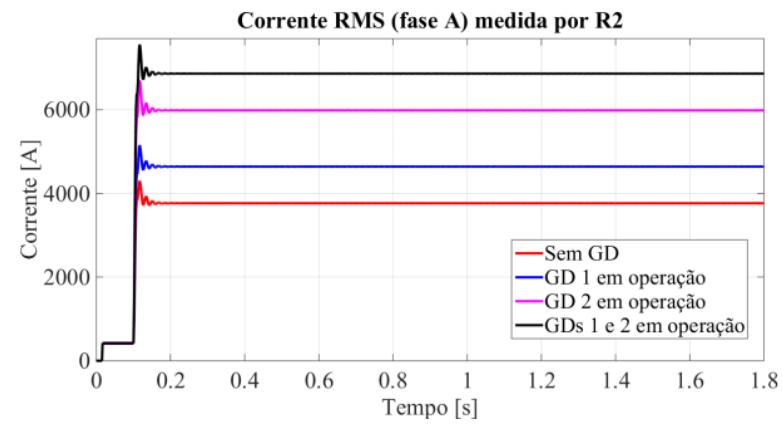

Fig 8. Corrente RMS na fase A medida por R2.

Nas tabelas deste trabalho, os valores de Iccmín e Iccmáx correspondem aos valores RMS das correntes de curtocircuito mínima (falta bifásica na barra 680) e máxima (falta trifásica no início da linha L632- 671) medidas no ponto de ocorrência da falta. Para o caso antes da inserção da GD no sistema, a Tabela 3 contém os tempos de atuação e margens de coordenação dos relés. 
Tabela 3. Caso sem GD

\begin{tabular}{|c|c|c|c|}
\hline Iccmín (A) & td R2 (s) & td R1 (s) & $\Delta$ t (s) \\
\hline 2378,22 & 0,392 & 1,145 & 0,753 \\
\hline Iccmáx (A) & & & \\
\hline 3749,71 & 0,153 & 0,454 & 0,301 \\
\hline
\end{tabular}

Observou-se que os relés R1 e R2 estão devidamente coordenados obedecendo aos critérios de seletividade, pois a menor margem de coordenação dos relés é 0,301 s.

\subsection{Caso com somente a GD 1 em operação}

A Tabela 4 contém os tempos de atuação e margens de coordenação dos relés no caso em que somente a GD 1 está em operação e não há uso do ajuste adaptativo para o relé R1.

Tabela 4. Com com a GD 1 em operação sem ajuste adaptativo de $R 1$

\begin{tabular}{|c|c|c|c|c|}
\hline Iccmín (A) & $\begin{array}{c}\text { td R2 } \\
(\mathbf{s})\end{array}$ & $\begin{array}{c}\text { td R1 } \\
(\mathbf{s})\end{array}$ & $\Delta \mathbf{t}(\mathbf{s})$ & $\begin{array}{c}\text { Dif. } \Delta \mathbf{t} \\
(\boldsymbol{\%})\end{array}$ \\
\hline 2703,59 & 0,3 & 0,872 & 0,572 & 24,04 \\
\hline Iccmáx (A) & & & & \\
\hline 4626 & 0,1 & 0,296 & 0,196 & 34,9 \\
\hline
\end{tabular}

Houve um aumento dos níveis de curto-circuito e redução nos tempos de atuação e margens de coordenação dos relés. Para a falta máxima, a margem de coordenação ficou abaixo do critério de segurança, o que prejudica a seletividade da proteção. O ajuste de R1 considerando somente a GD1 em operação foi calculado pelo bloco de controle utilizando os novos valores de correntes de falta, resultando em DT =0,23.

A Tabela 5 contém os tempos de atuação e margens de coordenação dos relés no caso em que a GD 1 está em operação e há uso do ajuste adaptativo no relé R1.

Tabela 5. Caso com a GD 1 em operação com ajuste adaptativo de $\mathbf{R} 1$

\begin{tabular}{|c|c|c|c|c|}
\hline Iccmín (A) & $\begin{array}{c}\text { td R2 } \\
(\mathbf{s})\end{array}$ & $\begin{array}{c}\text { td R1 } \\
(\mathbf{s})\end{array}$ & $\Delta \mathbf{t}(\mathbf{s})$ & $\begin{array}{c}\text { Dif. } \Delta \mathbf{t} \\
(\mathbf{\%})\end{array}$ \\
\hline 2703,59 & 0,3 & 1,18 & 0,88 & $-16,87$ \\
\hline Iccmáx (A) & & & & \\
\hline 4626 & 0,1 & 0,4 & 0,3 & 0,33 \\
\hline
\end{tabular}

É possível observar que, com a utilização do bloco de ajuste adaptativo, os tempos de atuação do relé R1 aumentam, o que faz com que as margens de coordenação aumentem e adquiram um valor mínimo igual a $0,3 \mathrm{~s}$, ou seja, dentro do critério de seletividade.

\subsection{Caso com somente a GD 2 em operação}

A Tabela 6 contém os tempos de atuação e margens de coordenação dos relés para o caso em que a GD 2 está em operação e não há uso do ajuste adaptativo para o relé R1.
Tabela 6. Caso com a GD 2 em operação sem ajuste adaptativo de $\mathbf{R} 1$

\begin{tabular}{|c|c|c|c|c|}
\hline Iccmín (A) & $\begin{array}{c}\text { td R2 } \\
(\mathbf{s})\end{array}$ & $\begin{array}{c}\text { td R1 } \\
(\mathbf{s})\end{array}$ & $\Delta \mathbf{t}(\mathbf{s})$ & $\begin{array}{c}\text { Dif. } \Delta \mathbf{t} \\
(\mathbf{\%})\end{array}$ \\
\hline 3107,71 & 0,225 & 1,595 & 1,37 & $-81,94$ \\
\hline Iccmáx (A) & & & & \\
\hline 5913,52 & 0,061 & 0,456 & 0,395 & $-31,23$ \\
\hline
\end{tabular}

Neste caso, as correntes de falta foram ainda maiores que no caso anterior (GD 1 em operação). No entanto, apenas R2 detectará a contribuição da GD e atuará em um tempo menor. Isso provoca um aumento nas margens de coordenação, o que faz com que os equipamentos do sistema fiquem submetidos por mais tempo a altos níveis de corrente, gerando perda de vida útil ou até danos mais críticos. Isso afeta a premissa de velocidade do sistema de proteção. Portanto, o ajuste de R1 considerando a GD na barra 632 deverá possuir um de dial de tempo menor. Este valor foi calculado pelo bloco de controle utilizando os novos valores de correntes de falta máxima e mínima e resultou em DT $=0,14$. A Tabela 7 contém os tempos de atuação e margens de coordenação dos relés para o caso com a GD 2 em operação e aplicação do ajuste adaptativo para o relé R1.

Tabela 7. Caso com a GD 2 em operação com ajuste adaptativo de $\mathbf{R} 1$

\begin{tabular}{|c|c|c|c|c|}
\hline Iccmín (A) & $\begin{array}{c}\text { td R2 } \\
(\mathbf{s})\end{array}$ & $\begin{array}{c}\text { td R1 } \\
(\mathbf{s})\end{array}$ & $\Delta \mathbf{t}(\mathbf{s})$ & $\begin{array}{c}\text { Dif. } \Delta \mathbf{t} \\
(\mathbf{\%})\end{array}$ \\
\hline 3107,71 & 0,225 & 1,31 & 1,085 & $-44,1$ \\
\hline Iccmáx (A) & & & & \\
\hline 5913,52 & 0,061 & 0,37 & 0,309 & $-2,66$ \\
\hline
\end{tabular}

Nota-se que, com a utilização do relé com ajuste adaptativo, os tempos de atuação do relé R1 diminuíram. Isso gerou uma redução nas margens de coordenação, o que significa que a atuação de retaguarda de $\mathrm{R} 1$ tornou-se mais rápida.

\subsection{Caso com as GDs 1 e 2 em operação}

A Tabela 8 contém os tempos de atuação e margens de coordenação, dos relés para o caso em que ambas as GDs 1 e 2 estão em operação e não há uso do ajuste adaptativo para o relé $\mathrm{R} 1$.

Tabela 8. Caso com as GDs 1 e 2 em operação sem ajuste adaptativo de $\mathbf{R} 1$

\begin{tabular}{|c|c|c|c|c|}
\hline Iccmín (A) & $\begin{array}{c}\text { td R2 } \\
(\mathbf{s})\end{array}$ & $\begin{array}{c}\text { td R1 } \\
(\mathbf{s})\end{array}$ & $\Delta \mathbf{t}(\mathbf{s})$ & $\begin{array}{c}\text { Dif. } \Delta \mathbf{t} \\
(\mathbf{\%})\end{array}$ \\
\hline 3341 & 0,194 & 0,882 & 0,68 & 9,69 \\
\hline Iccmáx (A) & & & & \\
\hline 6798 & 0,046 & 0,297 & 0,251 & 16,6 \\
\hline
\end{tabular}

Para este caso, as correntes de falta aumentaram ainda mais. Porém, o relé R1 detecta somente a contribuição da GD 1. Mesmo assim, ambos os relés operam em pontos de suas curvas onde a margem de coordenação entre eles torna-se 
menor do que o valor mínimo de segurança, o que significa que o sistema de proteção perde sua característica de seletividade. $\mathrm{O}$ ajuste de R1 considerando as GDs 1 e 2 em operação foi calculado pelo bloco de controle utilizando os novos valores de correntes de falta, resultando em DT $=0,2$.

A Tabela 9 contém os tempos de atuação e margens de coordenação, dos relés para o caso em que as GDs 1 e 2 estão em operação e há uso do ajuste adaptativo para o relé R1.

Tabela 9. Caso com as GDs 1 e 2 em operação com ajuste adaptativo de $\mathbf{R} 1$

\begin{tabular}{|c|c|c|c|c|}
\hline Iccmín (A) & $\begin{array}{c}\text { td R2 } \\
(\mathbf{s})\end{array}$ & $\begin{array}{c}\text { td R1 } \\
(\mathbf{s})\end{array}$ & $\Delta \mathbf{t}(\mathbf{s})$ & $\begin{array}{c}\text { Dif. } \Delta \mathbf{t} \\
(\mathbf{\%})\end{array}$ \\
\hline 3341 & 0,194 & 1,4 & 1,206 & $-4,8$ \\
\hline Iccmáx (A) & & & & \\
\hline 6798 & 0,046 & 0,349 & 0,303 & $-0,664$ \\
\hline
\end{tabular}

É possível observar que com o ajuste adaptativo, os tempos de atuação do relé R1 aumentam. Os relés passam a operar em pontos das curvas em que a margem de coordenação volta a ter valores iguais ou maiores que $0,3 \mathrm{~s}$, o que significa obedecer ao princípio da seletividade.

\section{CONCLUSÕES}

Neste trabalho, foi possível fazer análises a respeito da coordenação de relés de sobrecorrente no contexto do uso de geradores distribuídos nos sistemas de distribuição nos dias atuais. Desenvolveu-se um método simples de proteção adaptativa baseado no reajuste do dial de tempo do relé de acordo com os estados dos disjuntores das GDs. O método mostrou-se eficaz pois foi capaz de manter as margens de coordenação dos relés em níveis adequados para as diferentes topologias do sistema.

\section{AGRADECIMENTOS}

Agradecemos à Universidade Federal do Piauí (UFPI), ao Departamento de Engenharia Elétrica - UFPI e ao Programa de Pós-Graduação em Engenharia Elétrica - UFPI pela parceira e apoio ao desenvolvimento desta pesquisa.

\section{REFERÊNCIAS}

Britto, T. M. de; Moraes, D. R.; Marin, M. A. et al. (2004). Distributed generation impacts on the coordination of protection systems in distribution networks. Transmission and Distribution Conference and Exposition: Latin América, IEEE/PES, pp. 623-628.

Chaves, R. C. P. and Leão, F. B. (2014). Sistema de proteção adaptativo para redes de distribuição com inserção de geradores distribuídos. Cigré, Belo Horizonte, MG, Brasil.

Cheung, H.; Hamlyn, A.; WANG, L. et al. (2008). Networkintegrated adaptive protection for feeders with distributed generations. Power and Energy Society General Meeting - Conversion and Delivery of Electrical Energy in the 21st Century, IEEE., pp.1-8, 20-24 July.
Choi, J. H. et al. (2008). Adaptive protection schemes of distributed generation at distribution network for automatic reclosing and voltage sags. Sustainable Energy Technologies. Singapore: IEEE International Conference, pp. 810-815.

Dugan, R.C. and McDermott, T.E. (2002). Distributed generation. Industry Applications Magazine, IEEE vol.8, no.2, pp.19-25.

Filho, J. M. and Mamede, D. R. (2011). Proteção de Sistemas Elétricos de Potência. LTC, Rio de Janeiro, RJ.

KINDERMANN, G (2010). Curto-Circuito. 5a. ed. Florianópolis: Labplan, UFSC.

Luiz, C. M. (2012). Avaliação dos Impactos da Geração Distribuída para Proteção do Sistema Elétrico. Dissertação (Mestrado em Engenharia Elétrica) Universidade Federal de Minas Gerais (UFMG), Belo Horizonte.

Miguel, P. M. (2011). Introdução à Simulação de Relés de Proteção Usando a Linguagem "MODELS" do ATP. Rio de Janeiro: Ciência Moderna.

Rush, P. (2011). Proteção e Automação de Redes: Conceito e Aplicação ( $1^{a}$ ed.). São Paulo: Edgard Blücher.

Thong, V.V.; Driesen, J.; Belmans, R. (2007). Dispersed Generation Interconnection and Its Impact on Power Loss and Protection System. Universities Power Engineering Conference.

Yongli, Z.; Shaoqun, S.; Dewen, W.; (2009). Multiagentsbased wide area protection with best-effort adaptive strategy. International Journal of Electrical Power \& Energy Systems, Volume 31, Issues 2-3, FebruaryMarch, Pages 94-99.

Kersting, W. (1991). Radial distribution test feeders. Power Systems, IEEE Transactions on, vol. 6, no. 3, pp. 975985. 